\title{
Evaporative cooling of atomic chromium
}

\section{Citation}

Weinstein, Jonathan D., Robert deCarvalho, Cindy I. Hancox, and John M. Doyle. 2002.

"Evaporative Cooling of Atomic Chromium." Physical Review A 65 (2) (January 4). doi:10.1103/ physreva.65.021604.

\section{Published Version}

doi:10.1103/PhysRevA.65.021604

\section{Permanent link}

http://nrs.harvard.edu/urn-3:HUL.InstRepos:28422962

\section{Terms of Use}

This article was downloaded from Harvard University's DASH repository, and is made available under the terms and conditions applicable to Other Posted Material, as set forth at http:// nrs.harvard.edu/urn-3:HUL.InstRepos:dash.current.terms-of-use\#LAA

\section{Share Your Story}

The Harvard community has made this article openly available.

Please share how this access benefits you. Submit a story.

Accessibility 


\title{
Evaporative cooling of atomic chromium
}

\author{
Jonathan D. Weinstein, Robert deCarvalho, Cindy I. Hancox, and John M. Doyle \\ Department of Physics, Harvard University, Cambridge, Massachusetts 02138
}

(Received 8 July 2001; published 4 January 2002)

\begin{abstract}
We report the magnetic trapping and evaporative cooling of bosonic and fermionic isotopes of atomic chromium. Using a cryogenic helium buffer gas, $10^{12}$ chromium atoms are trapped at an initial temperature of $\sim 1 \mathrm{~K}$. The chromium atoms are then cooled adiabatically and evaporatively to temperatures as low as $\sim 10$ $\mathrm{mK}$. Elastic and inelastic ${ }^{52} \mathrm{Cr}$ collisional cross sections are measured over this temperature range. Prospects for simultaneously creating a ${ }^{52} \mathrm{Cr}$ Bose-Einstein condensate and ${ }^{53} \mathrm{Cr}$ Fermi degenerate gas will be discussed.
\end{abstract}

Evaporative cooling of magnetically trapped neutral atoms is a powerful, well-established technique for producing cold and dense atomic gases [1]. Having achieved notoriety from its use in the production of Bose-Einstein condensates [2], it has recently been extended to fermionic atoms and successfully used to create degenerate atomic Fermi gases [3].

Degenerate atomic Fermi gases are predicted to exhibit a wide variety of interesting phenomena, including modified collisional properties [4], changes in their light scattering properties, including linewidth narrowing [5], and Cooperpairing and superfluid phenomena [6]. All of these effects require, or are enhanced by, high particle number (which produces a high Fermi energy) and low temperature. We hope to achieve these conditions by evaporatively cooling boson and fermion isotopes of chromium to create a coexisting chromium Bose-Einstein condensate (BEC) and Fermi degenerate gas.

In a previous paper, we described the successful trapping of $\mathrm{Cr}$ through buffer-gas loading, a laser-free method for magnetically trapping large numbers of neutral atoms $[7,8]$. Here, we report the simultaneous trapping of boson and fermion isotopes of chromium, their evaporative cooling, and the measurement of $\mathrm{Cr}-\mathrm{Cr}$ collisional cross sections important for achieving quantum degeneracy.

The experimental apparatus is similar in design to our previous work [9]. The chromium atoms are still produced by laser ablation, cooled by a ${ }^{4} \mathrm{He}$ buffer gas inside by a dilution-refrigerator-cooled cell, and trapped by a 2.2-tesladeep magnetic "anti-Helmholtz" quadrupole trap. The copper cell used in the previous experiment is replaced by a double-walled plastic cell with a superfluid liquid helium jacket to provide thermal conductivity [10]. Based on a design from the MIT hydrogen BEC experiment [2,11], this electrically insulating cell allows the magnetic trapping fields to be changed rapidly without inducing eddy currents, preventing undue heating.

The trapped atoms are probed by absorption spectroscopy on the $a^{7} S_{3} \rightarrow z{ }^{7} P_{2,3}$ and 4 transitions at $\sim 430 \mathrm{~nm}[12,13]$. We determine the number and temperature of the trapped atoms by fitting the observed $\mathrm{Cr}$ spectrum to the calculated spectrum of a Boltzmann distribution of atoms inside the trap.

Using buffer-gas loading, we initially trap $10^{12} m_{J}=+3$ $\mathrm{Cr}$ atoms at a peak density approaching $10^{13} \mathrm{~cm}^{-3}$ and a temperature of $\sim 1 \mathrm{~K}$. While there are other $m_{J}$ states (where $m_{J}$ is the projection of the total electron angular momentum $J$ along the field axis) initially present in the trap, they are more weakly confined by the trap and are quickly lost due to evaporation. Within $10 \mathrm{~s}$, we pump out the helium buffer gas by cooling the cell to a temperature below $200 \mathrm{mK}$, liquefying the ${ }^{4} \mathrm{He}$ onto the cell walls. By that time, inelastic spinchanging collisions between the $\mathrm{Cr}$ atoms have reduced the number of trapped atoms to $\sim 5 \times 10^{11}$.

In addition to the dominant chromium isotope ${ }^{52} \mathrm{Cr}$ (a boson, with nuclear spin $I=0$ ), we also trap ${ }^{50} \mathrm{Cr}$ (boson, $I$ $=0$ ) and ${ }^{53} \mathrm{Cr}$ (fermion, $I=\frac{3}{2}$ ) atoms, as shown in Fig. 1 $[13,14]$. In the trap, we observe the ${ }^{53} \mathrm{Cr}$ only in the $\mid m_{J}=$ $\left.+3, m_{I}=+\frac{3}{2}\right\rangle$ fully polarized "stretched state." The other hyperfine states of ${ }^{53} \mathrm{Cr}$ are lost on a time scale faster than we are able to observe $(<20 \mathrm{~s})$. This rapid loss is likely due to spin-exchange collisions, which are expected to dominate all other inelastic loss processes for atoms not in a fully polarized state $[15,16]$.

The ${ }^{50} \mathrm{Cr}:{ }^{52} \mathrm{Cr}$ ratio in the trap is (within our experimental error) equal to the ratio of the natural isotopic abundances [17]. Surprisingly, the ${ }^{53} \mathrm{Cr}:{ }^{52} \mathrm{Cr}$ ratio is also approximately equal to the ratio of the natural abundances. It is assumed

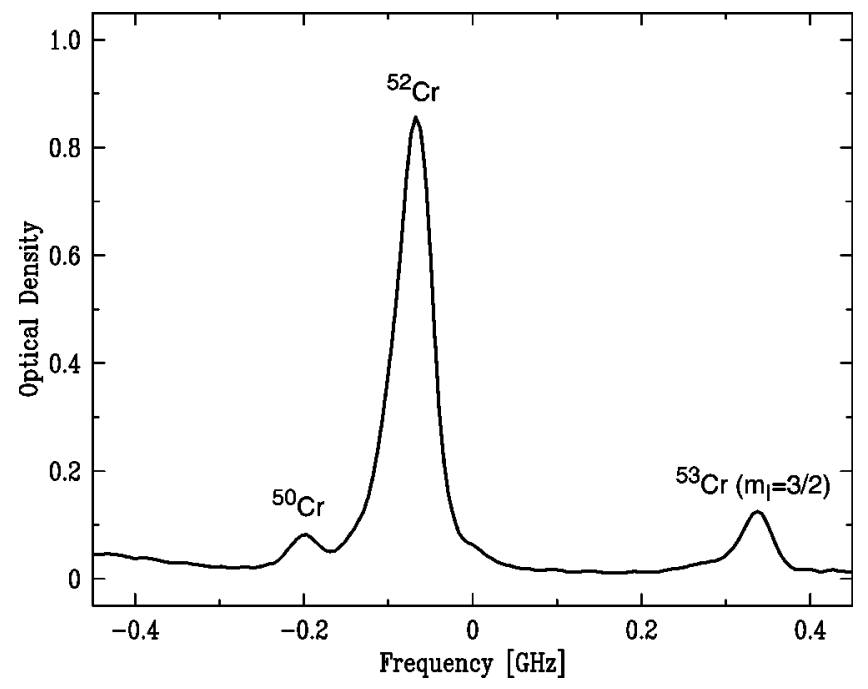

FIG. 1. Absorption spectrum of trapped $\mathrm{Cr}$ on the $a^{7} S_{3}$ $\rightarrow z{ }^{7} P_{3}$ transition. The $A=50,52$, and 53 isotopes can be clearly resolved and appear in their approximate natural abundances. Trap depth $0.7 \mathrm{~K}$, number $2 \times 10^{11}$, temperature $100 \mathrm{mK}$. 


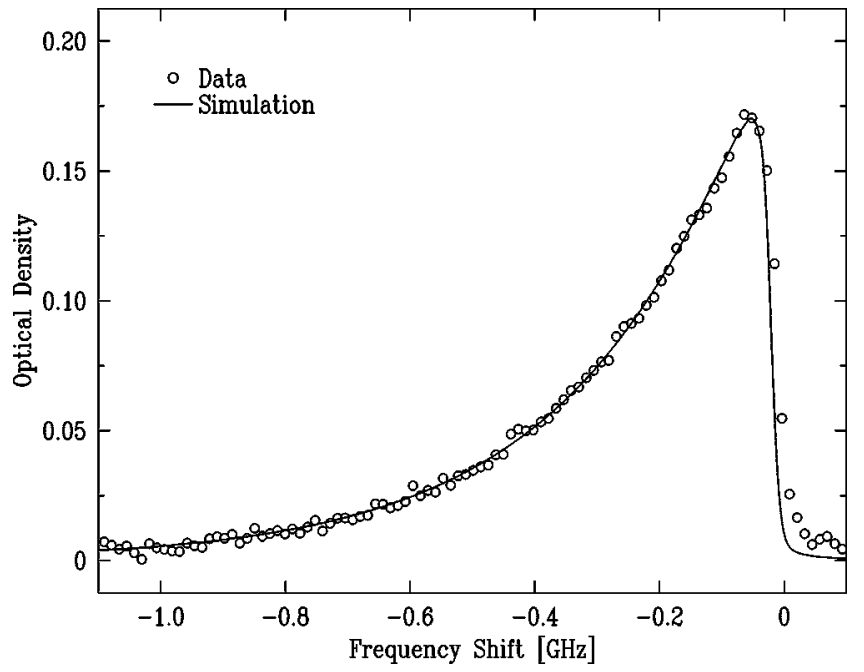

FIG. 2. A spectrum of trapped ${ }^{52} \mathrm{Cr}$ on the $a{ }^{7} S_{3} \rightarrow z{ }^{7} P_{2}$ transition, fit to the simulated spectrum of a Boltzmann distribution of $9 \times 10^{10}$ atoms in the trap at $55 \mathrm{mK}$ and peak density 5 $\times 10^{10} \mathrm{~cm}^{-3}$. The dominant broadening mechanism is Zeeman broadening due to the inhomogeneous magnetic field of our trap. Frequency shift is plotted relative to the field-free line. The number and temperature of the atoms and the frequency shift zero are the only free parameters in this fit.

that due to the high-energy nature of the ablation process, all $m_{J}$ and $m_{I}$ states are initially produced in equal number. Hence, we would naively expect the population of any individual $m_{I}$ state to be reduced by a factor of $(2 I+1)$. It is possible that spin-exchange processes inside the trap may play some role by preferentially enhancing the population of the $\left|m_{J}=+3, m_{I}=+\frac{3}{2}\right\rangle$ state at the expense of other levels.

Once trapped, the atoms are cooled by reducing the trapping fields. Two cooling mechanisms are involved. First, the reduction in confinement cools the atoms through adiabatic expansion while keeping their phase-space density constant. Second, the loss of atoms over the trap edge cools the chromium through evaporative cooling, typically resulting in an increase in phase-space density. A typical spectrum of cooled chromium is shown in Fig. 2.

We observe that as we lower the trapping fields, the phase-space density initially increases, but makes only modest gains. At lower temperatures $(\sim 10 \mathrm{mK})$, we find we are unable to further increase the phase-space density through evaporative cooling. We attribute this loss to poor $\mathrm{Cr}-\mathrm{Cr}$ collisional cross sections. Elastic collisions (which thermalize the distribution of atoms) allow for evaporative cooling. Inelastic spin-changing collisions cause trap loss and heating. The efficacy of evaporative cooling depends crucially on the ratio of the elastic to inelastic collision rates [16].

To measure the rate of inelastic collisions, we monitor the decay of atoms in the trap and fit it to the expected functional form for two-body loss $\left(\dot{n}=-\Gamma_{\mathrm{in}} n^{2}\right)$, as shown in Fig. 3. The accuracy of the fit indicates that $\mathrm{Cr}-\mathrm{Cr}$ collisions are the dominant cause of atom loss. If there is trap loss due to collisions with background ${ }^{4} \mathrm{He}$ gas, it occurs on a time scale longer than $200 \mathrm{~s}$, indicating a negligible effect on evaporative cooling in our experiment.

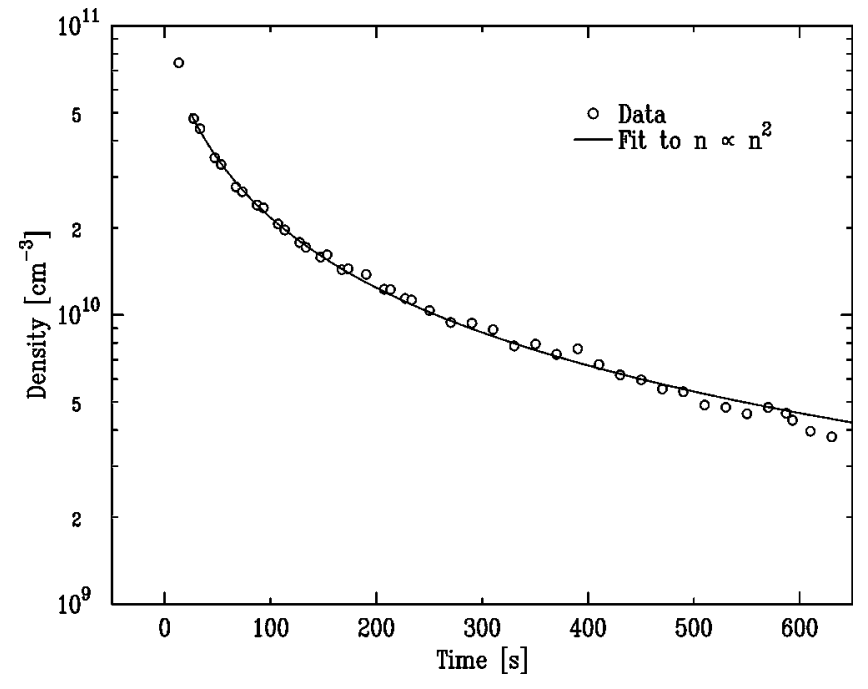

FIG. 3. The peak chromium density as a function of time in a 0.2-K-deep trap. Fit to the expected functional form for loss due to $\mathrm{Cr}-\mathrm{Cr}$ collisions.

To determine the rate of elastic collisions, we selectively optically pump atoms out of the trap to perturb the trap population from thermal equilibrium. We then observe the reequilibration of the atoms, as shown in Fig. 4. The elastic

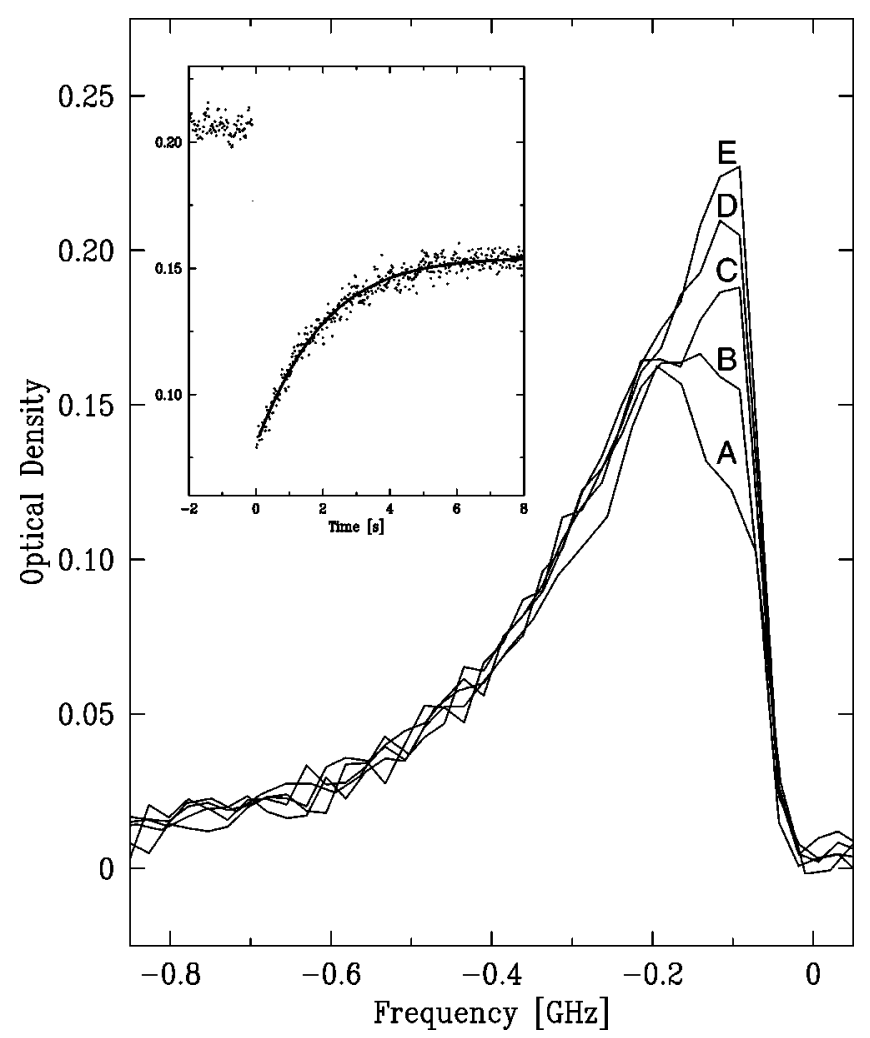

FIG. 4. Typical optical pumping data. The spectra $A-E$ were taken at 1-s intervals following the optical pumping (the pumping beam was tuned to $-0.12 \mathrm{GHz}$ ). The inset displays the optical density at the pumping frequency as a function of time, fit to the expected functional form. 


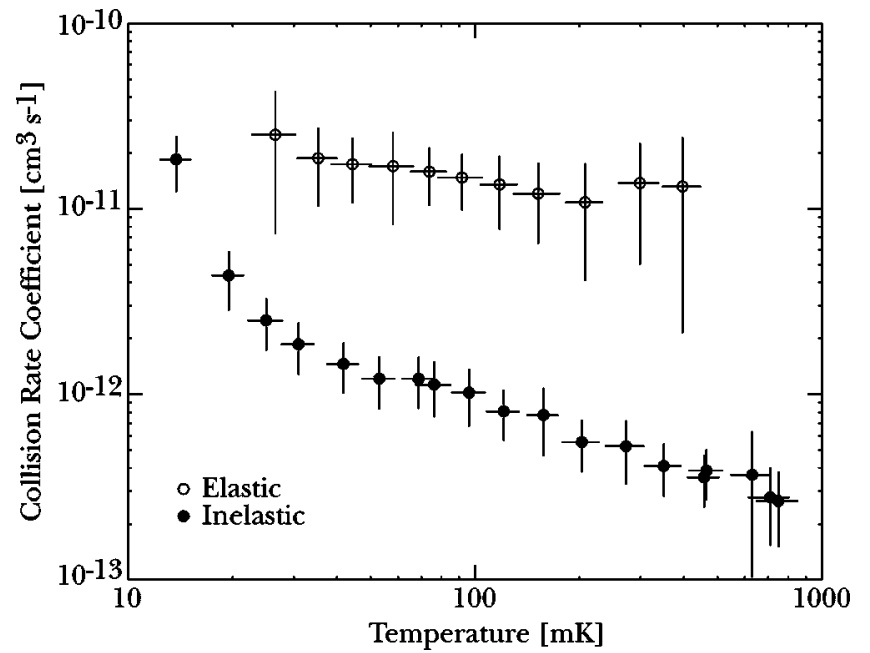

FIG. 5. ${ }^{52} \mathrm{Cr}-{ }^{52} \mathrm{Cr}$ collision rate coefficients plotted as a function of atom temperature. The error bars include both statistical error and estimates of our systematic error. It should be noted that the magnetic fields encountered by the atoms scale as the temperature. We were unable to measure ${ }^{53} \mathrm{Cr}$ collision rates, but could determine that the ${ }^{53} \mathrm{Cr}-{ }^{52} \mathrm{Cr}$ inelastic collision rate was less than or equal to the ${ }^{52} \mathrm{Cr}-{ }^{52} \mathrm{Cr}$ rate.

collision rate is determined from the equilibration rate and a simple model of the equilibration process [18].

An independent method of determining the ratio of the elastic to inelastic collision rates is used to confirm these measurements. Because elastic and inelastic collisions provide competing cooling and heating mechanisms, their ratio determines the equilibrium temperature of the atom cloud. We measure this temperature (inside the known trapping potential) and combine this result with a model of the trap dynamics to extract the collision ratio $[19,20]$. We find that the observed equilibrium temperatures are consistent with the measured ratios of the collision rates to within the quoted error bars.

The measured collision rate coefficients (both elastic and inelastic) are shown in Fig. 5 as a function of temperature. At the higher initial temperatures, the ratio of elastic to inelastic collision rates is large enough to permit the phase-space density to be increased through evaporative cooling. However, at lower temperatures, the inelastic collision rate rapidly increases, making evaporative cooling inefficient.

In addition to the crucial $\mathrm{Cr}-\mathrm{Cr}$ scattering properties described above, we also measured $\mathrm{Cr}$-He collisional properties. By observing the exponential decay of atoms inside the trap with the cell walls at elevated temperatures (and hence large densities of helium within the cell), we are also able to measure inelastic $\mathrm{He}^{52} \mathrm{Cr}$ collisional cross sections for temperatures near $1 \mathrm{~K}$. With ${ }^{3} \mathrm{He}$ buffer gas, we find an inelastic collision rate constant of $\Gamma_{\text {in }}=(2 \pm 1) \times 10^{-18} \mathrm{~cm}^{3} \mathrm{~s}^{-1}$. Due to details of the method of measurement, we were less sensitive to ${ }^{4} \mathrm{He}-{ }^{52} \mathrm{Cr}$ inelastic collisions, and can only place an upper limit of $\Gamma_{\text {in }}<10^{-16} \mathrm{~cm}^{3} \mathrm{~s}^{-1}$.

We also determined the elastic ${ }^{52} \mathrm{Cr}-\mathrm{He}$ cross section. This was done by measuring the diffusion of $\mathrm{Cr}$ through the helium buffer gas in the absence of the magnetic trap at $T$ $\sim 1 \mathrm{~K}[21]$. With ${ }^{4} \mathrm{He}$ buffer gas, we find an elastic collision cross section of $\sigma_{\mathrm{el}}=1 \times 10^{-14} \mathrm{~cm}^{2}$ at $600 \mathrm{mK}$. However, because of uncertainties in the quantity of ${ }^{4} \mathrm{He}$ buffer gas, this number is only accurate to within an order of magnitude. With ${ }^{3} \mathrm{He}$ buffer gas, we measure an elastic collision cross section of $\sigma_{\mathrm{el}}=(1.8 \pm 0.6) \times 10^{-14} \mathrm{~cm}^{2}$ at $300 \mathrm{mK}$.

In conclusion, we magnetically trapped multiple chromium isotopes in large number and evaporatively cooled them. At low temperatures, evaporative cooling was limited by a large and increasing inelastic collision rate. The overall large scale of the trap loss could be due to either chromium's large magnetic moment or a strong spin-orbit coupling arising in the $\mathrm{Cr}-\mathrm{Cr}$ molecular potential. The considerable increase in the inelastic collision rate coefficient (also accompanied by an increasing elastic collision cross section) observed as the chromium is cooled is likely due to the presence of a shape resonance [22,23]. Over the temperature range encountered in this work, chromium is not in the ultracold limit: many higher-order partial waves contribute to the scattering process. The presence of a shape resonance (or "pseudobound state") in higher-order partial waves would lead to an increase in the inelastic collision rate. Theory is currently unable to conclusively identify the physical mechanisms behind this inelastic collision rate; the $\mathrm{Cr}-\mathrm{Cr}$ interatomic potential is not understood well enough, unlike that of hydrogen and the alkali-metal atoms.

If the observed scattering rates persist at lower temperatures, their ratio will be too small to support efficient evaporative cooling. However, if the large inelastic rate is due to a sufficiently narrow shape resonance, as discussed above, or other "high-temperature" processes, the loss rate might decrease at lower temperatures and allow continued evaporative cooling. As accurate theoretical predictions are currently unavailable in this regime, further experimentation is necessary to discover these ultracold cross sections.

Because one of our goals is to reach quantum degeneracy with large numbers of atoms, if the ratio of elastic to inelastic collisions does indeed increase at sub-mK temperatures, we would like to reach those temperatures at high densities (to provide the collisions for continued evaporative cooling) and with large numbers of atoms remaining.

One method for getting through the $10 \mathrm{mK}$ "problem region" may be to laser Doppler cool trapped ${ }^{52} \mathrm{Cr}$ on the $\left|{ }^{7} S_{3}, m_{J}=3\right\rangle \rightarrow\left|{ }^{7} P_{4}, m_{J}=4\right\rangle$ transition [24-26]. With the appropriate experimental setup, it should be possible to laser precool the trapped $\mathrm{Cr}$ atoms on a reasonable time scale without significant loss. If the ultracold collision cross sections are greatly improved relative to the higher-temperature values, it should be possible to create a chromium BEC and degenerate Fermi gas using further evaporative cooling.

We would like to thank Dima Egorov for experimental assistance and for assistance in the construction of our trap magnet. We would like to thank A. Dalgarno, H. R. Sadeghpour, Paul S. Julienne, Carl J. Williams, and John L. Bohm for discussions concerning $\mathrm{Cr}-\mathrm{Cr}$ collisions. This work was supported by the National Science Foundation. 
[1] Harald F. Hess, Phys. Rev. B 34, 3476 (1986).

[2] M. H. Anderson et al., Science 269, 198 (1995); K. B. Davis et al., Phys. Rev. Lett. 75, 3969 (1995); C. C. Bradley, C. A. Sackett, and R. G. Hulet, ibid. 78, 985 (1997); D. G. Fried et al., ibid. 81, 3811 (1998).

[3] B. DeMarco and D. S. Jin, Science 285, 1703 (1999); Andrew G. Truscott et al., ibid. 291, 2570 (2001); F. Schreck et al., Phys. Rev. A 64, 011402(R) (2001).

[4] B. DeMarco, S. B. Papp, and D. S. Jin, Phys. Rev. Lett. 86, 5409 (2001).

[5] K. Helmerson, M. Xiao, and D. Pritchard, IQEC'90 book of abstracts; Th. Busch, J. R. Anglin, J. I. Cirac, and P. Zoller, Europhys. Lett. 44, 1 (1998); J. Ruostekoski and J. Javanainen, Phys. Rev. Lett. 82, 4741 (1999).

[6] H. T. C. Stoof, M. Houbiers, C. A. Sackett, and R. G. Hulet, Phys. Rev. Lett. 76, 10 (1996); G. Bruun, Y. Castin, R. Dum, and K. Burnett, Eur. Phys. J. D 7, 433 (1999); E. Timmermans, K. Furuya, P. W. Milonni, and A. K. Kerman, e-print cond-mat/0103327.

[7] J. D. Weinstein, R. deCarvalho, J. Kim, D. Patterson, B. Friedrich, and J. M. Doyle, Phys. Rev. A 57, R3173 (1998).

[8] R. deCarvalho et al., Eur. Phys. J. D 7, 289 (1999).

[9] J. Kim et al., Phys. Rev. Lett. 78, 3665 (1997).

[10] J. D. Weinstein, Ph.D. thesis, Harvard University, 2001.

[11] D. G. Fried, Ph.D. thesis, Massachusetts Institute of Technology, 1999.

[12] C. E. Moore, Atomic Energy Levels as Derived from the Analyses of Optical Spectra, Volume II (National Bureau of Standards, Washington, D.C., 1971).

[13] T. Reinhardt, J. Maichel, M. Baumann, and J. Krüger, Z. Phys.
D: At., Mol. Clusters 34, 87 (1995).

[14] K. Heilig and D. Wendlandt, Phys. Lett. 25A, 277 (1967).

[15] H. T. C. Stoof, J. M. V. A. Koelman, and B. J. Verhaar, Phys. Rev. B 38, 4688 (1988).

[16] W. Ketterle and N. J. Van Druten, Adv. At., Mol., Opt. Phys. 37, 181 (1996).

[17] J. R. Parrington, H. D. Knox, S. L. Breneman, E. M. Baum, and F. Feiner, Chart of the Nuclides, 15th ed. (General Electric Co., San Jose, 1996).

[18] The equilibration model is based on the assumption that the distribution of the atom cloud is unperturbed from thermal equilibrium except in the narrow region of optical pumping. The rate that atoms are replenished in this region is then found from a detailed balance argument and the assumption that a single elastic collision is sufficient to scatter an atom in the region of optical pumping out of it.

[19] J. M. Doyle, J. C. Sandberg, I. A. Yu, C. L. Cesar, D. Kleppner, and T. J. Greytak, Physica B 194-196, 13 (1994).

[20] R. deCarvalho, Ph.D. thesis, Harvard University, 2002.

[21] J. B. Hasted, Physics of Atomic Collisions, 2nd ed. (Elsevier, New York, 1972).

[22] H. M. J. M. Boesten, C. C. Tsai, B. J. Verhaar, and D. J. Heinzen, Phys. Rev. Lett. 77, 5194 (1996).

[23] B. DeMarco, J. L. Bohn, J. P. Burke, Jr., M. Holland, and D. S. Jin, Phys. Rev. Lett. 82, 4208 (1999).

[24] C. C. Bradley, J. J. McClelland, W. R. Anderson, and R. J. Celotta, Phys. Rev. A 61, 053407 (2000).

[25] A. S. Bell, et al., Europhys. Lett. 45, 156 (1999).

[26] K. Helmerson, A. Martin, and D. E. Pritchard, J. Opt. Soc. Am. B 9, 1988 (1992). 